\section{References}

1 Wald N, Cuckle HS, Harwood CA. Screening for open neural tube defects in England and Wales. Br Med F 1979; ;i:331.

${ }^{2}$ Chamberlain J. Human benefits and costs of a national screening programme for neural tube defects. Lancet 1978;ii:1293-6.

3 Tunkel V. Abortion: how early, how late, and how legal? $\mathrm{Br} M e d f$ 1979 ;ii :253-6.

4 Anonymous. No case for an abortion Bill. Br Med $\mathcal{F} 1979$;ii:230.

5 Russell W. Abortion bill in overdrive. Br Med $\mathcal{F} 1979$;ii: 1452.

${ }^{6}$ North-east Thames Regional Health Authority. Regional prenatal screening service. London, May 1979.

7 Clarke PC, Gordon YB, Kitau MJ, Chard T, Letchworth AT. Screening for foetal neural tube defects by maternal plasma alpha-foeto-protein determination. Br $\mathcal{F}$ Obstet Gynaec 1977;84:563-73.

${ }^{8}$ Clarke M. Feasibility of serum- $\alpha$-fetoprotein screening for fetal neural tube defects. Lancet $1977 ; \mathrm{i}: 1098-9$.

${ }^{9}$ Heward JA, Clarke M. Communications to an antenatal clinic. $\mathrm{Br} \mathrm{Med} \mathcal{F}$ 1976;i:1201-4.
${ }^{10}$ Holland M. Towards healthy babies: the prevention of cerebral palsy. London: Spastics Society, 1979:14-5.

${ }^{11}$ General Register Office. Census 1951-Classification of occupations. London: HMSO, 1956.

12 Report of the Committee on Child Health Services. Fit for the future. London: HMSO, 1976. (Court Report.)

13 British Paediatric Association/Royal College of Obstetricians and Gynaecologists Liaison Committee. Recommendations for the improvement of infant care during the perinatal period in the United Kingdom. London: BPA/RCOG, 1978.

${ }^{14}$ Department of Health and Social Security/Child Poverty Action Group. Reaching the consumer in the ante-natal and child health services. London: DHSS-CPAG, 1978: 34-5.

${ }^{15}$ Pembrey $M$. Why Corrie's Bill must give prenatal tests more leeway. Guardian $1980 \mathrm{Feb} 1: 12$ (col 1).

(Accepted 26 February 1980)

\title{
Prospective controlled trial comparing colostomy irrigation with "spontaneous-action" method
}

\author{
N S WILLIAMS, D JOHNSTON
}

\section{Summary and conclusions}

Thirty randomly selected patients with permanent colostomies entered a prospective controlled trial comparing colostomy irrigation with spontaneous action. Each patient was interviewed and examined before irrigation was begun and again after the technique had been used for three months. Each then reverted to spontaneous action for a further three months and was then reassessed.

Eight patients abandoned irrigation and $22(73 \%)$ adhered to the protocol. Irrigation caused no mishaps or complications. The mean time spent managing the stoma was $45 \pm S E M 9 \mathrm{~min} / 24$ hours during spontaneous action and $53 \pm 9 \mathrm{~min} / 24$ hours during irrigation. This difference was not significant. The numbers of bowel actions weekly were $13 \pm$ SEM 2 during spontaneous action and $6 \pm 1$ during irrigation $(p<0.01)$. Irrigation reduced odour and flatus in 20 patients and enabled 12 out of 18 to stop using drugs and seven to discard their appliance. Irrigation also improved the social life of 18 patients and the working conditions of eight out of 14 .

These findings show that some patients may not be suitable for irrigation but that for many it is better than the conventional British method of colostomy management. With modern apparatus the technique is safe.

\section{Introduction}

The best way of managing a colostomy at home is debatable. In Britain the most commonly used method is "spontaneous action," which means that by dietary manipulation and the use of drugs the colostomy is induced to act once or twice daily

\footnotetext{
University Department of Surgery, General Infirmary, Leeds LS1 3EX

N S WILLIAMS, MB, FRCS, lecturer in surgery

D JOHNSTON, CHM, FRCS, professor of surgery
}

at predictable times. ${ }^{1}$ Fewer than half of all patients achieve this ideal, however, ${ }^{2} 3$ and about $60 \%$ have difficulties with their appliance owing to skin allergy, soreness, leakage, odour, and flatus. ${ }^{2}$

The alternative method is colostomy irrigation, which is popular in America and reportedly eliminates many of the problems associated with natural evacuation. ${ }^{4-6}$ There is no record of a controlled clinical comparison of the two methods, however, and we therefore decided to conduct such a trial.

\section{Patients and methods}

Twenty-four men and six women aged 17-78 years (mean 58.4 years) were admitted to the trial. Each had had a permanent colostomy constructed after abdominoperineal excision of the rectum a mean of $6 \cdot 3$ years before and were using spontaneous action as the only method of colostomy care. The patients were selected at random from those attending for routine follow-up at a rectal clinic. The only contraindications to entry to the trial were lack of bathroom facilities, severe locomotor handicaps, or stenosis of the colostomy.

Patients were interviewed at the beginning of the trial and any abnormality of the stoma recorded. Blood was taken for a full blood count and measurement of serum urea and electrolyte concentrations. They were then taught the irrigation method and instructed to use it for three months, at first daily but reducing the number of irrigations at their discretion. After three months the patients were interviewed and examined and the blood investigations repeated. Patients then reverted to the spontaneous-action method for a further three months and were then reassessed. Throughout the trial each patient recorded the number of bowel actions daily, the time spent managing the stoma, and the frequency of irrigation.

Irrigation technique-Figure 1 shows the components of the cone-type irrigation set. The flow-control clamp is closed and the reservoir filled with tepid water. The clamp is opened to fill the tubing with water, then closed again, and the reservoir is suspended above the shoulder, the patient being seated on the toilet. The drainage sleeve is next secured round the patient's waist, so that its upper end lies around the stoma and its lower end hangs between the legs into the bowl (fig 2). The cone is then lubricated and inserted into the stoma, but only far enough to allow the irrigating fluid to flow into the intestine without escaping. The flow-control clamp is then opened and $250-500 \mathrm{ml}$ fluid allowed to flow into the colon. 
After several minutes the cone is withdrawn and evacuation of colonic contents allowed to occur.

\section{Results}

Of the 30 patients who entered the trial, $22(73 \%)$ adhered to the protocol. The remaining eight abandoned irrigation during the three months, three because of aesthetic objections and five owing to technical difficulties, particularly inability to introduce the cone satisfactorily into the stoma. Only the 22 patients who adhered to the trial protocol are included in the analysis.
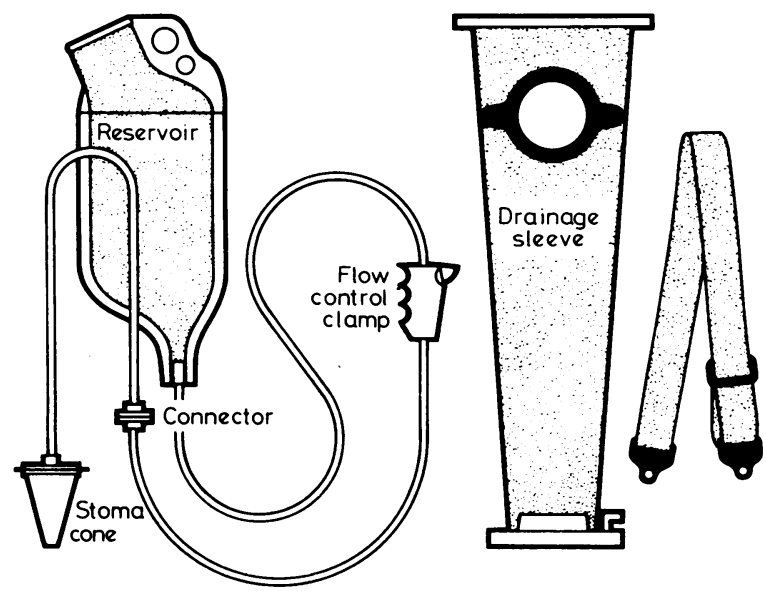

FIG 1-Equipment used for irrigation.

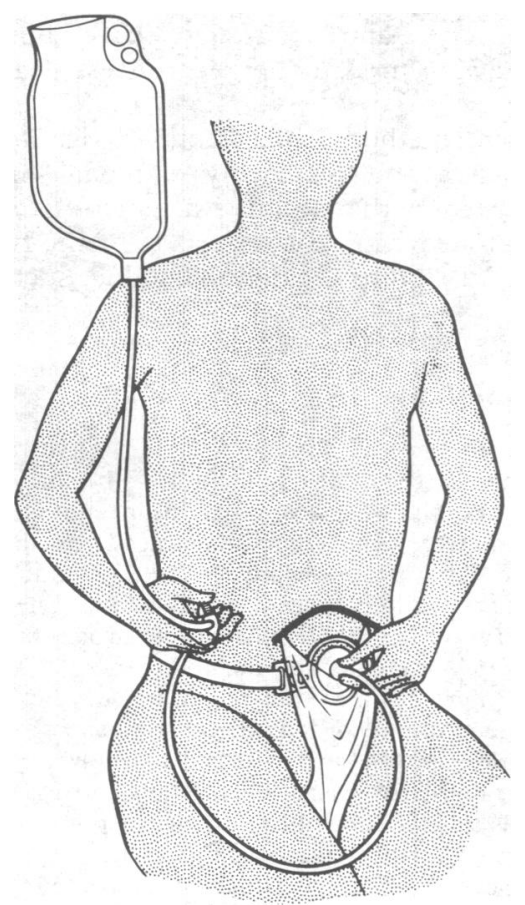

FIG 2-Drainage sleeve in position and cone inserted into stoma. Flow-control clamp is opened, allowing fluid to enter colon.

Time spent managing the colostomy-Figure 3 shows for each patient the time spent daily managing the colostomy. The mean time expended during spontaneous action was $45 \pm S E M 9 \mathrm{~min} / 24$ hours and during irrigation $53 \pm 9 \mathrm{~min} / 24$ hours. This difference was not significant.

Frequency of colostomy actions-With the outcome of each irrigation counted as one bowel action (fig 4), the mean number of colostomy actions weekly while using irrigation was $6 \pm$ SEM 1 and while using spontaneous action $13 \pm 2$ ( $p<0.01$; Student's $t$ test for paired data).

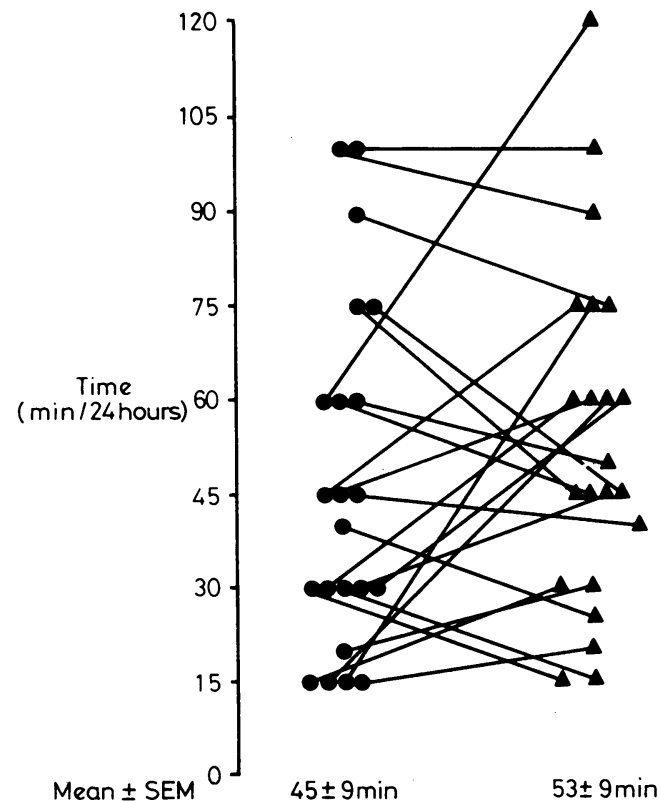

FIG 3-Time spent managing stoma/24 hours during spontaneous action $(O)$ and irrigation $(\boldsymbol{A}) .(45 \pm 9 \mathrm{~min}$ v $53 \pm 9 \mathrm{~min}$ : not significant.)

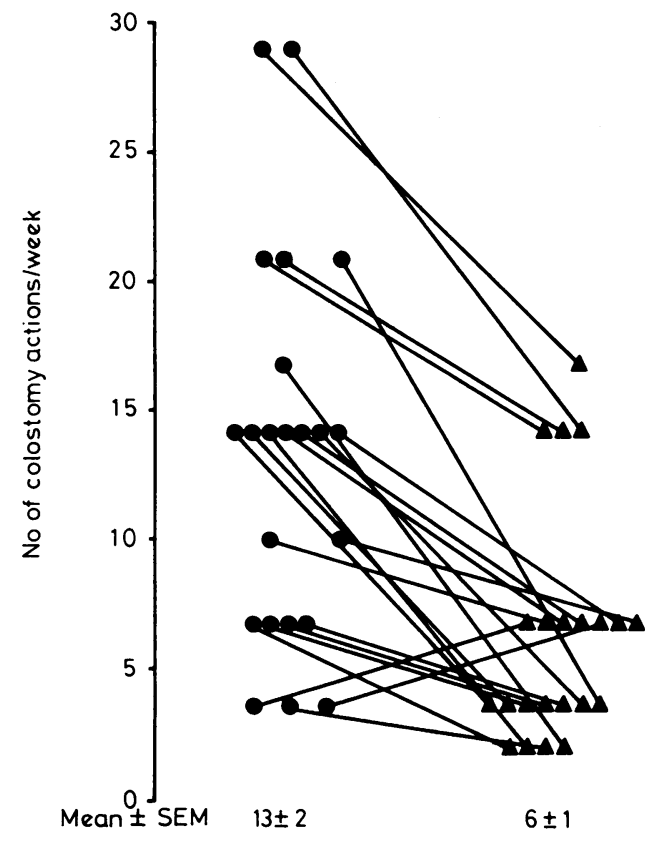

FIG 4-Numbers of bowel actions weekly during spontaneous action (O) and irrigation ( $\mathbf{A})$. Outcome of each irrigation counted as one bowel action; thus during use of irrigation method very few spontaneous bowel actions occurred. $(13 \pm 2$ actions $v \quad 6 \pm 1$ actions: $\mathrm{p}<0.01$.)

Frequency of irrigation-Eleven patients irrigated daily, seven irrigated on alternate days, and four irrigated every third day. Of the 11 patients who irrigated daily, four had occasional faecal actions between irrigations, but each of these patients had had 21 or more actions a week while using spontaneous action. Four patients in the group who irrigated less often complained of mucous discharge between irrigations. Thus of the 22 patients, 14 had no colostomy actions whatever between irrigations and seven were confident enough to discard their appliance and rely merely on a small pad to cover the stoma.

Odour and flatus-The amount of odour and flatus from the colostomy was assessed simply by asking the patient at the end of the 
spontaneous-action period whether it had increased, decreased, or not changed compared with the irrigation period. Twenty patients noticed an increase, none noticed a decrease, and two noticed no change.

Diet-Sixteen patients had eliminated two or more items from their diet while using spontaneous action in an attempt to control their colostomy action. During the irrigation period 10 patients could reintroduce these items into their diet, only to eliminate them again when they reverted to spontaneous action.

Medication-At the beginning of the trial 18 patients were relying on drugs to control their colostomy action. Twelve of them stopped using drugs during irrigation but had to reintroduce them when they reverted to spontaneous action.

Employment-Eighteen patients had been in full-time employment before operation, and 14 returned to work after operation, 10 to the same job and four to part-time or lighter occupations. None of the 14 patients found that irrigation interfered with their jobs, whereas eight complained of difficulties at work (frequent changes of the appliance or anxiety about leakage) while using spontaneous action.

Social life-Eighteen patients stated that their social life was improved while using irrigation. They were more confident in meeting people and visiting public places, especially restaurants. Four had gone swimming, which they would not have contemplated while using spontaneous action.

Complications-Irrigation was not associated with any major complication; in particular, no patient suffered colonic perforation or water retention. There was no significant difference in full blood count and serum urea and electrolyte concentrations after three months of irrigation when compared with these measurements after the same period of spontaneous action. At the start of the trial three patients had prolapsing colostomies and two paracolostomy hernias. None of them found irrigation difficult, and it did not exacerbate their original stoma problem.

Irrigation or spontaneous action?-At the final visit the patients were asked which method of colostomy care they would use in future. Seven stated that, although irrigation was useful, they intended to use it only on special occasions, and 15 intended to continue using irrigation regularly; 13 of them were still using the technique when interviewed three months later.

\section{Discussion}

Colostomy irrigation was first described in Britain in 1927.7 It received enthusiastic support ${ }^{8}{ }^{9}$ until 1945, when Gabriel ${ }^{10}$ reported nine cases of colonic perforation that resulted in eight deaths. Subsequently irrigation was condemned ${ }^{11}$ and, with a few notable exceptions, ${ }^{45}$ spontaneous action remained the conventional method of stoma care in most centres. In America, on the other hand, most surgeons, stoma therapists, and patients have continued to favour irrigation since the introduction of the closed-system method advocated by Brinkley. ${ }^{12}$

With the equipment then available, it is not surprising that perforation of the colon was a common problem when irrigation was first introduced. The recommendation was to use a thick rubber tube and insert this as far as possible into the colon. ${ }^{13}$ During the past decade there has been much improvement in the design of equipment for colostomy irrigation, and colonic perforation is now unlikely with the cone type of equipment. None of our patients suffered this complication.

The other reason why irrigation was originally unpopular in Britain was the poor standard of domestic sanitation. In 1950 nearly $40 \%$ of households lacked a fixed bath, $8 \%$ had neither an inside nor outside toilet, and only $10 \%$ had central heating. In 1978 , however, $90 \%$ of households had the sole use of all basic amenities, and over half had central heating. ${ }^{14}$

Many patients with colostomies are dissatisfied with the spontaneous-action method. Grier $e t a^{2}$ and later Devlin et $a l^{3}$ found that some $60 \%$ of their patients had three or more actions daily, and the same proportion of patients had problems with leakage, excoriation, and odour and flatus, which seriously interfered with their social lives and employment. Many of our patients had similar problems, though only nine had more than three actions a day when using the spontaneous method.
Although irrigation was unacceptable to eight patients, in the remaining 22 it improved their everyday lives and wellbeing. Irrigation significantly reduced the number of colostomy actions and enabled some patients to dispense with their appliance altogether. It also decreased flatus and odour from the colostomy and allowed some patients to eat a normal diet and some to dispense with medication. These advantages encouraged 15 patients to replace spontaneous action with irrigation as the sole method of colostomy care. Although seven patients intended to use the technique only occasionally, all agreed that it had advantages. In particular, it allowed them to fulfil social commitments that they would not have contemplated while using spontaneous action.

Patients of less intelligence or in whom there are technical difficulties, particularly stenosis of the colostomy, should probably not be offered the technique. Such patients, however, are in the minority, and provided patients are introduced to irrigation at an early stage most should adapt to its use. In America the patient is taught the method a few days after operation. In our view, however, the optimum time is about three months after operation. This allows bowel function to settle and gives the perineal wound time to heal, making irrigation more comfortable.

Initially the irrigation should be performed once every 24 hours. When a stool-free period has been established the interval between irrigations may be increased gradually to 48 hours or even to 72 hours in some patients.

The daily instillation of $500 \mathrm{ml}$ or more of tap water into the colon might be expected to cause water retention. This did not occur in our trial, however, and there was no significant change in serum urea and electrolyte concentrations after three months of irrigation compared with these measurements after the same period of spontaneous action. Nevertheless, irrigation should probably not be recommended to patients with evidence of renal or cardiac impairment.

Our results show that some patients may not be suitable for irrigation but that for many it is a better technique than the conventional British method of colostomy management. Each patient deserves individual consideration. A flexible and common-sense approach to colostomy care is required rather than rigid adherence to any single regimen. If more patients are taught the irrigation method they can choose the method that suits them best.

We thank Abbott Laboratories Ltd, Queenborough, Kent, for providing the equipment, and Mrs Elaine Nutter and Miss Lynne Harrison for secretarial work.

Requests for reprints should be sent to Mr N S Williams.

\section{References}

1 Anonymous. Living with a colostomy. Br Med f 1957;i:274

2 Grier WRN, Postel AH, Jyarse A, Localio SA. An evaluation of colonic stoma management. Surg Gynecol Obstet 1964;118:1234-42.

${ }^{3}$ Devlin HB, Plant JA, Griffin M. Aftermath of surgery for anorectal cancer. $\mathrm{Br}$ Med F 1971 ;iii:413-8.

4 Griffith DA, Philpotts E, Espiner HS, Eltringham WK. The continent colostomy. Gut 1976;17:385-402.

5 Seargeant PW. Colostomy management by the irrigation technique: review of 165 cases. $\mathrm{Br}$ Med $\mathcal{F} 1966$;ii:25-6.

${ }^{6}$ Mazier P, Dignan D, Smith DG. Effective colostomy irrigation. Surg Gynecol Obstet 1976;142:905-9.

7 Lockhart-Mummery P. Discussion on colostomy. Proc $R$ Soc Med $1927 ; 20: 1461$

${ }^{8}$ Gabriel WB. Discussion on colostomy. Proc $R$ Soc Med 1927;20:1452-66.

9 Gabriel WB, Lloyd Davies OV. Colostomy. Br f Surg 1935;22:520-2.

${ }_{10}$ Gabriel WB. Discussion of the management of the permanent colostomy. Proc $R$ Soc Med 1945;38:692-4.

11 Anonymous. Colostomy and the patient. Br Med f 1958;i:448.

12 Brinkley GE. Construction and care of abdominal colostomy. Am $\mathcal{f}$ Surg $1952 ; 83: 807-12$

${ }^{13}$ Gordon Watson C. Discussion on the management of the permanent colostomy. Proc $R$ Soc Med 1945;38:692-4.

14 Central Statistical Office. Social trends. No 10. London: HMSO, 1980. 